\title{
FASCITIS NECROTIZANTE. EXPERIENCIA EN LOS ÚLTIMOS CINCO AÑOS EN UN SERVICIO DE CIRUGÍA ORTOPÉDICA Y TRAUMATOLOGÍA.
}

\author{
Autores: Aguilar JC 1, Cuñat BR 2, Angulo MA 1, Baeza J 1, Amaya V 1, Baixauli FF \\ ${ }^{1}$ Hospital Univeristari I Politècnic La Fe, Valencia, Spain \\ ${ }^{2}$ Hospital de Manises, Valencia, Spain.
}

\section{Resumen:}

La fascitis necrotizante es una infección rara, muy agresiva y potencialmente letal. Identificarla a tiempo supone un reto y es clave para poder realizar una intervención quirúrgica precoz y mejorar la supervivencia.

El objetivo del trabajo es analizar el manejo de esta patología en un servicio de Cirugía Ortopédica y Traumatología para optimizar resultados.

Estudio retrospectivo observacional que incluye ocho pacientes tratados de fascitis necrotizante entre los años 2013-2018. Revisión bibliográfica comparando datos de la evidencia actual con los obtenidos en la serie.

Se intervinieron 8 pacientes en 5 años. La mediana de edad fue de 60,5 años. El 75\% fueron varones. La localización más frecuente fue en miembros inferiores. El $88 \%$ de los casos presentaba factores de riesgo, destacando la historia de traumatismo o agresión cutáneo-mucosa reciente y diabetes. La prueba diagnóstica más utilizada fue la tomografía computerizada con una sensibilidad 100\%. La media del score "Laboratory Risk Index for Necrotizing Fasciitis" fue 7,71/13.

El 75\% de los pacientes fue operado en menos de 24 horas. Se re-intervinieron el 88\%, el $43 \%$ antes de 24 horas desde la primera cirugía.

Se utilizó terapia de presión negativa con buenos resultados en un $25 \%$ de los casos. La mortalidad intrahospitalaria fue del 25\% y la media de seguimiento 260,5 días.

Para mejorar la supervivencia en la fascitis necrotizante es clave el diagnóstico precoz, que sólo es posible si identificamos el prototipo de paciente de riesgo y conocemos las herramientas diagnósticas disponibles además de la clínica.

Palabras clave: Infecciones de partes blandas. Fascitis necrotizante. Infecciones necrotizantes de partes blandas. Necrotizing fasciitis. Soft-tissue infections. Necrotizing soft-tissue infections. 


\section{INTRODUCCIÓN}

La infección de partes blandas es muy frecuente, su tratamiento es conservador y en la gran mayoría de los casos no requiere de la valoración de un especialista en Cirugía Ortopédica y Traumatología. Sin embargo, existe un subgrupo mucho menos frecuente al que denominamos infección necrotizante de partes blandas (INPB). Este grupo ha recibido diferentes nombres a lo largo de la historia, siendo actualmente el término de fascitis necrotizante (FN) el que se tiende a utilizar de manera consensuada para evitar confusiones. La característica más importante de la FN es su agresividad. Se trata de una infección que progresa muy rápidamente causando necrosis tisular y una grave repercusión sistémica que condiciona su elevada mortalidad. El tratamiento debe ser de entrada quirúrgico, ya que la cirugía precoz ha demostrado mejorar la supervivencia (1). Cuando nos enfrentamos a un caso clínico concreto es muy difícil diferenciar en el momento inicial si realmente nos encontramos ante una infección necrotizante. Por lo tanto, aspectos esenciales como son el diagnóstico y tratamiento precoz se convierten en un gran reto.

¿Cómo puedo ser capaz de identificar una fascitis necrotizante a tiempo para mejorar la supervivencia? El objetivo del trabajo es analizar el manejo de la FN en un servicio de Cirugía Ortopédica y Traumatología (COT) con el fin de optimizar los resultados y aportar una visión práctica para el manejo de esta letal enfermedad.

Clásicamente se han diferenciado dos grupos: la tipo I, infección polimicrobiana en la que habitualmente están involucadros al menos un germen anaerobio y uno o más anerobios facultativos y/o enterobacterias; $y$ la tipo II, monomicrobiana, causada por estreptococos beta-hemolíticos del grupo A, siendo el más frecuente el Streptococcus Pyogenes, aunque también otros grupos de estreptococos o Staphylococcus Aureus. (3, 7).

\section{MATERIAL Y MÉTODOS}

$\mathrm{Se}$ realiza un estudio retrospectivo observacional monocéntrico en el que se incluyen los pacientes con diagnóstico de FN tratado quirúrgicamente por el servicio de COT entre los años 2013 y 2018. Se obtiene la serie de pacientes mediante búsqueda informática en la base de datos de informes quirúrgicos del hospital. Se acota la búsqueda a las hojas quirúrgicas de Cirugía Ortopédica y Traumatología exclusivamente, dentro de las fechas indicadas. Los términos de búsqueda utilizados son "fascitis necrotizante" y "fascitis necrosante". Para esta búsqueda se utiliza la herramienta de explotación de la información del software "Orion". Los criterios de exclusión establecidos fueron la ausencia de cultivos microbiológicos positivos que confirmaran el diagnóstico de FN.

De cada paciente se recogen múltiples variables: Datos epidemiológicos básicos como la edad y el sexo; la presencia de factores de riesgo para el desarrollo de FN entre los que incluimos: diabetes mellitus (DM), enfermedad vascular periférica, inmunodepresión, e historia de traumatismo y/o agresión cutáneo-mucosa reciente. Se define reciente el period inferior a 3 semanas, considerando que dicho tiempo coincide con el de cicatrización y restitución de las lesiones de partes blandas. Nos referimos a traumatismo como cualquier agresión ("blunt trauma") distinta de las agresiones cutáneo-mucosas. En el grupo de agresiones cutáneo-mucosas se incluyen cualquier solución de continuidad de dichas barreras, incluidos los procesos quirúrgicos. Otras variables recogidas son la localización de la infección; variables clínicas iniciales incluyendo temperatura, ausencia o presencia de fiebre, hipotensión y shock; variables analíticas iniciales incluyendo los parámetros para el cálculo del score: "Laboratory Risk Indicator for Necrotizing Fasciitis" (LRINEC) (2); y pruebas de imagen utilizadas ya sea ecografía, tomografía computerizada (TC) o resonancia magnética 
(RM). Se recogen asimismo los tiempos desde inicio de síntomas hasta primera cirugía, tiempo desde primera cirugía hasta re-intervención, el número de reintervenciones y el resultado de los cultivos $y$ tipo de FN.

IMÁGENES CLÍNICAS cultivos, requerimientos de procesos de tratamiento especiales y complicaciones incluyendo la mortalidad intrahospitalaria se expresan en porcentaje. Para el cálculo del score LRINEC se utiliza la puntuación propia de la escala (2) y se
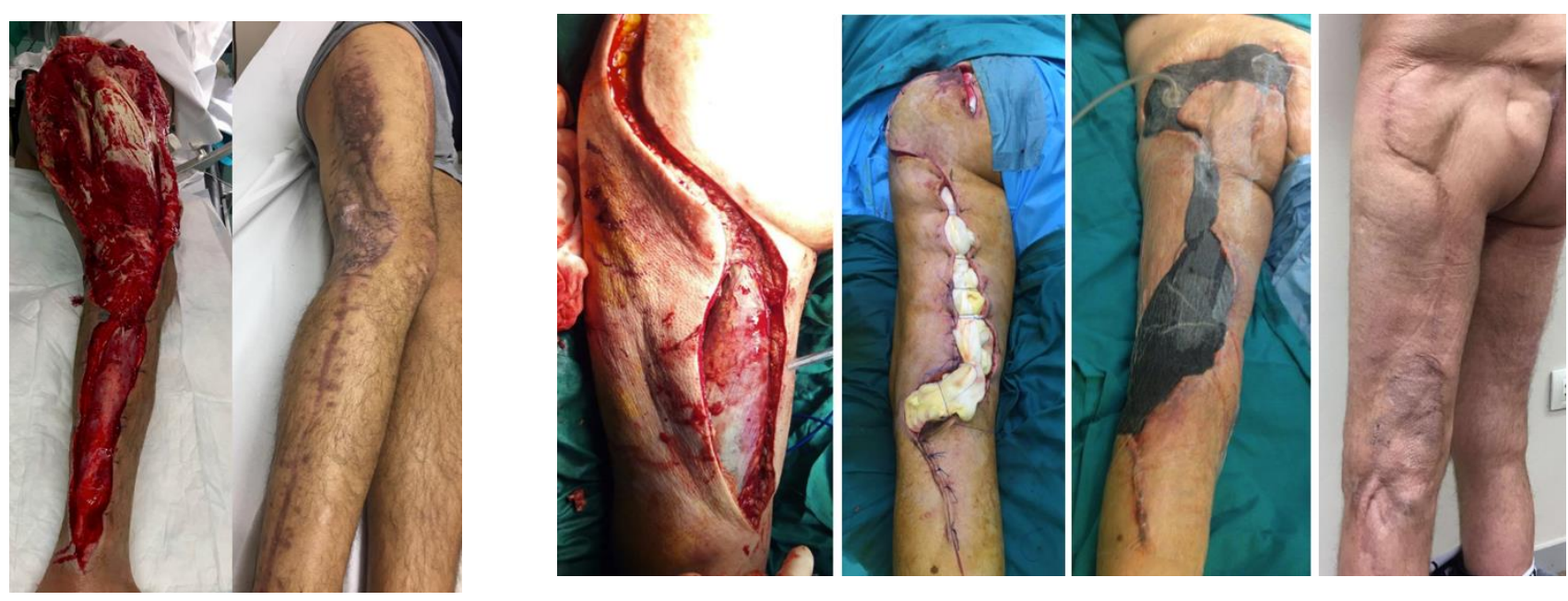

Por último, se recogen aspectos quirúrgicos incluyendo el requerimiento de amputación o cirugía de cobertura de partes blandas; utilización terapia de presión negativa; y finalmente el tiempo de seguimiento en días. Todos los datos son recogidos y tratados por un investigador diferente al cirujano implicado en el tratamiento de cada caso. Los pacientes fueron intervenidos por distintos cirujanos pertenecientes a diferentes unidades dentro de un mismo servicio y seguidos al alta hospitalaria en consultas externas.

Se realiza una revisión bibliográfica actualizada en la base de datos Pubmed utilizando para la búsqueda las palabras clave "fascitis necrotizante" y "necrotizing fasciitis" y descartando todo artículo no escrito en castellano o en inglés, para realizar una comparación de los datos con los obtenidos en nuestra serie.

Para los estadísticos descriptivos se utiliza la mediana para la edad y el sexo se expresa en porcentaje. Las demás variables incluyendo los factores de riesgo, localización de la infección, realización de pruebas de imagen, tipo de FN en base a resultados de los expresa como la media. En cuanto a los tiempos de intervención se establecen dos categorías según sea mayor o menor de 24 horas, siendo este el límite entre intervención precoz o diferida descrito por la bibliografía $(1,3,4)$. También se establecen dos categorías para expresar los signos clínicos que se miden en porcentaje según su presencia o ausencia.

\section{RESULTADOS}

Se realiza tratamiento quirúrgico de un total de 8 pacientes con diagnóstico de FN durante un periodo de 5 años, entre 2013 y 2018. El $75 \%$ de los pacientes fueron de sexo masculino. La mediana de edad fue de 60,5 años. La localización más frecuente de la infección fue en miembros inferiores, representando el $100 \%$ de los casos. (Tabla 1) La media de seguimiento de los pacientes fue de 260 días.

En cuanto a las comorbilidades y factores de riesgo para el desarrollo de $\mathrm{FN}$, todos los 
pacientes presentaban algún factor de riesgo clásico. En este sentido lo más frecuente fue la presencia de traumatismo previo o agresión cutáneo-mucosa reciente incluyendo cirugía, apareciendo en 7 del total de los 8 pacientes de la serie, lo que supone un porcentaje de un $88 \%$ (Tabla 1). De estos pacientes, un $43 \%$ tenía antecedente de intervención quirúrgica reciente, entre las que se incluyeron: tratamiento de una fractura abierta tipo IIIC mediante fijador externo y bypass vascular, extirpación de un neuroblastoma de pelvis e intervención de un sinus pilonidal (estas dos últimas intervenciones realizadas por las especialidades correspondientes). Otro $43 \%$ de los pacientes presentó otra agresión cutáneo-mucosa reciente distinta de cirugía, incluyendo complicación de hemorroides, brote de hidrosadenitis supurativa e inyección intramuscular. El restante 14\% tuvo historia de traumatismo previo reciente sin agresión cutáneo-mucosa ("blunt trauma"), que consistió en una fractura de ramas pélvicas (Figura 1).

TRAUMATISMO O AGRESIÓN CUTÁNEO MUCOSA INCLUYENDO CIRUGÍA $(\mathrm{N}=7)$



\section{FIGURA 1}

El otro factor de riesgo más frecuente fue la DM que estaba presente en el $62,5 \%$ de los pacientes. Todos los pacientes diabéticos presentaban diabetes tipo II no insulinodependiente. Otros factores de riesgo que aparecieron con menor frecuencia fueron la inmunodepresión, presente en un $38 \%$ de los pacientes. Entre las causas de inmunosupresión aparecen: SIDA, neuroblastoma con afectación de la médula ósea, y leucemia mieloide aguda con aplasia medular. El último factor de riesgo que aparece en nuestros pacientes es la enfermedad vascular periférica, presente en un $13 \%$ de los pacientes (Tabla 1). Es destacable que en la mayoría de los pacientes $(75 \%)$ coexistían dos o más factores de riesgo de los mencionados.

En cuanto a las variables clínicas registradas sólo un $25 \%$ de los pacientes presentaron fiebre, un $13 \%$ hipotensión y otro 13\% desarrollaron un shock séptico en las primeras 24 horas. La media del score LRINEC (2) fue de 7,71 sobre 13 puntos, aunque sólo fue realizado en 7 pacientes por falta de datos analíticos en el octavo paciente.

TABLA 1

\begin{tabular}{|c|c|c|c|c|c|c|c|c|}
\hline \multirow[t]{2}{*}{$\mathrm{N}$} & \multirow[t]{2}{*}{$\overline{\mathrm{EDA}} \mathrm{D}$} & \multirow[t]{2}{*}{ SEXO } & \multirow{2}{*}{$\begin{array}{l}\text { SEGUIMIENTOS } \\
\text { (días) }\end{array}$} & \multirow[t]{2}{*}{ LOCALIZACION } & \multicolumn{4}{|c|}{ FACTORES RIESGO FN } \\
\hline & & & & & TRAUMATISMO* & $\mathrm{DM}^{\mathrm{d}}$ & IDP $^{f}$ & $\mathrm{EVPh}^{\mathrm{h}}$ \\
\hline 1 & 24 & M & 266 & MII $^{\mathbf{a}}$ & $\begin{array}{c}\mathrm{SI}\left(\mathrm{cx}^{\mathrm{c}}\right. \\
\text { neuroblastoma) }\end{array}$ & NO & $\begin{array}{c}\text { SI } \\
\text { (neuroblastoma) }\end{array}$ & NO \\
\hline 2 & 30 & $\mathbf{M}$ & 686 & MID $^{\mathbf{b}}$ & $\begin{array}{c}\text { SI (fr. abierta + } \\
\text { bypass) }\end{array}$ & NO & NO & NO \\
\hline 3 & 42 & M & 26 & MII & SI (inyección droga) & NO & SI (SIDA) & NO \\
\hline 4 & 59 & $\mathbf{M}$ & $\begin{array}{c}<7 \\
\text { (EXITUS) }\end{array}$ & MID & SI (cx hemorroides) & DM2 $^{\mathrm{e}}$ & SI $\left(\mathbf{L M A}^{\mathrm{g}}\right)$ & NO \\
\hline 5 & 60 & $\mathbf{M}$ & 405 & MID & SI (cx sinus pilonidal) & DM2 $^{\mathrm{e}}$ & NO & SI \\
\hline 6 & 65 & $\mathbf{F}$ & 78 & MII & $\begin{array}{c}\text { SI (brote } \\
\text { hidrosadenitis) }\end{array}$ & DM2 $^{\mathrm{e}}$ & NO & NO \\
\hline 7 & 77 & $\mathbf{F}$ & 102 & MII & SI (fr. ramas pélvicas) & DM2 $^{\mathrm{e}}$ & NO & NO \\
\hline 8 & 79 & $\mathbf{M}$ & $\begin{array}{c}<7 \\
\text { (EXITUS) }\end{array}$ & MID & NO & DM2 $^{\mathrm{e}}$ & NO & NO \\
\hline
\end{tabular}


${ }^{a}$ MII: Miembro inferior izquierdo; ${ }^{b}$ MID: Miembro inferior derecho; ${ }^{c} \boldsymbol{C X}$ : Cirugía; ${ }^{d}$ DM: Diabetes mellitus; ${ }^{e}$ DM2: Diabetes mellitus tipo 2 (no insulinodependiente); ${ }^{f}$ IDP: Inmunodepresión; ${ }^{g}$ LMA: Leucemia mieloide aguda; ${ }^{h}$ EVP: Enfermedad vascular periférica

* Traumatismo o agresión cutáneo mucosa reciente incluyendo cirugía

En casi todos los pacientes (88\%) se realizó alguna prueba de imagen. Todo paciente en que se realizó prueba de imagen incluyó TC, cuya sensibilidad diagnóstica fue del $100 \%$. Otras pruebas menos utilizadas fueron la ecografía (25\%) y la RM (13\%).

Respecto a los tiempos de realización del tratamiento quirúrgico, en el $75 \%$ de los pacientes se realizó la intervención en las primeras 24 horas desde el inicio de los síntomas. Se realizó re-intervención en quirófano del $88 \%$ de los pacientes y el $43 \%$ de estos se re-intervinieron en 24 horas desde la cirugía inicial. En base a los resultados de los cultivos intraoperatorios, el $25 \%$ fueron FN tipo II y el $75 \%$ tipo I; siendo lo más frecuente en este último grupo la aparición de especies de Clostridium, representando un $38 \%$ del total.

En total, sólo 1 paciente fue amputado $(13 \%)$, un $38 \%$ requirió técnicas de reconstrucción de partes blandas basadas en colgajos y realizados por el servicio de Cirugía Plástica, y en un $25 \%$ de los casos se utilizó terapia de presión negativa.

La mortalidad intrahospitalaria fue del $25 \%$ y del resto de pacientes el seguimiento medio total fue de 304 días, siendo 78 días el menor tiempo de seguimiento.

\section{DISCUSIÓN}

La mortalidad de la FN es muy elevada tal y como han descrito algunos estudios $(1,5)$ y así sucede en nuestra serie. También los datos epidemiológicos son concordantes con lo publicado hasta la fecha ya que lo más frecuente es la afectación de varones en edad media y de localización en miembros inferiores (6) por lo que el patrón de afectación sigue la corriente habitual.

En cuanto al tipo de FN según microorganismo causante, clásicamente se han diferenciado dos grupos distintos. La FN tipo I es una infección polimicrobiana en la que habitualmente están involucadros al menos un germen anaerobio y uno o más anerobios facultativos $\mathrm{y} / \mathrm{o}$ enterobacterias. La FN tipo II es monomicrobiana y está causada por estreptococos beta-hemolíticos del grupo $A$, siendo el más frecuente el Streptococcus Pyogenes, aunque también puede estar causada por otros grupos de estreptococos o por Staphylococcus Aureus. $(3,7)$.

La incidencia de los tipos I y || suele ser similar (8) aunque varía en function de las series. En nuestra serie es más frecuente el tipo I, pero nos llama la atención sobre manera la importante representación por especies de Clostridium, hasta un 38\%, cuando es un microorganismo poco frecuente en lo publicado en la bilbiografía $(6,9)$. Este dato cobra importancia cuando tenemos en cuenta que, además del tratamiento quirúrgico, la instauración de tratamiento antibiótico es fundamental en el manejo.

Si atendemos a los datos clínicos de los pacientes, la aparición de dolor es una constante, siendo el síntoma principal, como es bien conocido. Sin embargo, los signos sistémicos sugestivos de sepsis como la hipotensión, el shock y la fiebre no siempre aparecen inicialmente y así lo hemos visto en nuestra serie. Estos signos han demostrado tener una baja sensibilidad que oscila entre el $10-40 \%$ según estudios (3). Estos datos subrayan la importancia de no infravalorar la gravedad de los pacientes en la fase temprana de la enfermedad por el hecho de no presentar alteración de constantes vitales. Al analizar los antecedentes de los pacientes de nuestra serie destaca la presencia de factores de riesgo para el desarrollo de $\mathrm{FN}$ en un elevado porcentaje de los casos (88\%), sobre todo la presencia de traumatismos o agresión cutáneomucosa reciente incluyendo cirugía, y la DM. La DM ha sido descrita en la literatura como un factor de riesgo importante para presentar FN, e incluso como un factor de mal pronóstico clínico que puede predecir la necesidad de amputación (10); sin embargo, 
en muchos trabajos no se especifica el tipo de DM. Todos los pacientes diabéticos en nuestra serie eran DM tipo II, lo cual coincide con aquellos estudios que especifican el tipo de DM (11-14).

En muchos de los casos, en un mismo sujeto existía acumulación de más de dos factores de riesgo simultáneos. Creemos que es importante conocer estos factores para estar alerta y pensar en el diagnóstico de $\mathrm{FN}$ al encontrarnos ante un caso con este tipo de antecedentes y un cuadro clínico sospechoso.

A la hora de apoyar el diagnóstico de sospecha de $\mathrm{FN}$ se utilizan comúnmente pruebas de imagen, y se han propuesto varias en esta patología, como la radiografía, ecografía, TC O RM. Quizá destaca de manera negativa la RM por tratarse de una prueba con poca disponibilidad de urgencia y que puede retrasar el diagnóstico. En cuanto a pruebas más rápidas y accesibles, la radiografía presenta una baja sensibilidad ya que es capaz de detectar gas, pero éste no siempre aparece en los tejidos ya que es dependiente del microorganismo causante. El TC es una prueba que presenta una alta sensibilidad, tal y como ha demostrado en nuestra serie, habiendo sido capaz de diagnosticar a la totalidad de los pacientes. En nuestro ámbito, la realización de TC no supone un retraso en el tratamiento debido a la rápida accesibilidad, pero no en todos los centros sucede así. Es necesario destacar que no siempre son necesarias pruebas de imagen para decidir intervenir a un paciente con un cuadro clínico sospechoso de FN, y nunca si va a suponer un retraso en el tratamiento.

¿Qué herramientas diagnósticas rápidas disponemos entonces para apoyar nuestro diagnóstico clínico y tomar la decisión de realizar una intervención quirúrgica? Se han propuesto valores analíticos que son capaces de diferenciar entre infecciones de partes blandas necrotizantes y no necrotizantes, destacando el score LRINEC (2) que reúne una serie de parámetros analíticos sanguíneos (nivel de Proteína $C$ Reactiva, leucocitos, hemoglobina, sodio, creatinina y glucosa) para crear una puntuación de 0 a 13 y estratificar a los pacientes en perfiles de bajo, intermedio o alto riesgo para la detección precoz de la patología. Desde que se propuso el score se ha utilizado en varios trabajos que han intentado validarlo. Una revisión reciente (8) que incluyó siete estudios que utilizaron el score LRINEC ha propuesto un punto de corte de 5,8/13. A partir de ese valor o mayor, el valor predictivo positivo para FN oscila entre $57 \%$ hasta $92 \%$ en tres estudios diferentes incluidos, y el valor predictivo negativo oscila entre $86 \%$ y $96 \%$ en otros dos estudios incluidos en la revisión.

Por lo tanto, si seguimos este punto de corte, ante un score menor, sería muy improbable la presencia de $\mathrm{FN}$ ya que es una prueba con un alto valor predictivo negativo, y por lo tanto la podríamos utilizar como screening. Si bien en nuestra serie la media de LRINEC de los 7 pacientes en los que se realizó fue de 7,71/13, nos impresiona que hasta en 2 pacientes el score estaba por debajo del punto de corte propuesto. Aunque hablamos de una serie pequeña, en nuestro caso no hubiera sido útil el score LRINEC como screening si se hubiera utilizado en el momento del diagnóstico, ya que hasta un $28 \%$ de los pacientes no se hubieran diagnosticado, con las consecuencias que ello conlleva.

Para profundizar en este tema, analizamos a los 2 pacientes con el score más bajo y pudimos comprobar que eran pacientes inmunodeprimidos con afectación de médula ósea y aplasia medular $y$, por lo tanto, un recuento bajo de leucocitos, que es uno de los parámetros que más puntúa en el test. Creemos por lo tanto que este score puede no ser útil en este tipo de pacientes, que a su vez son un grupo con mayor riesgo de presentar la patología.

En cuanto al tratamiento, y en vistas a los tiempos de intervención, se intentaron respetar los principios básicos para mejorar la supervivencia siempre que fue posible: hasta en un $75 \%$ de los casos se realizó intervención en las primeras 24 horas desde el inicio de los síntomas. En contraste, al analizar los tiempos a la hora de realizar una re-intervención quirúrgica, vimos que tan sólo un $43 \%$ de los casos se re-intervinieron en menos de 24 horas desde la primera 
cirugía (Figura 2). Se ha destacado la importancia de realizar una reevaluación quirúrgica a las 24 horas de la primera cirugía y después tantas veces como sea necesario hasta conseguir el control local de la infección $(3,4)$. Aunque nuestros resultados son buenos si atendemos a la mortalidad intrahospitalaria y requerimiento de amputación, hay que destacar que se pueden optimizar los tiempos de reintervención y para esto es importante tener en cuenta que cuando se presenta un paciente con $\mathrm{FN}$ requiere una planificación para disponer de quirófanos y recursos, personal, etc. A pesar de la importancia de los tiempos de intervención para el aumento de supervivencia, no se suelen reflejar datos concretos en este sentido en los trabajos publicados, y creemos necesario que aparezcan más estudios con este enfoque para destacar con cifras la importancia de intervenir y re-intervenir a los pacientes de forma precoz.

En los casos en los que se utilizó la terapia de presión negativa, siempre tras el control local de la infección, los resultados fueron satisfactorios, acorde a lo publicado en la literatura, aunque con series de casos pequeñas $(15,16)$.

\section{FIGURA 2}
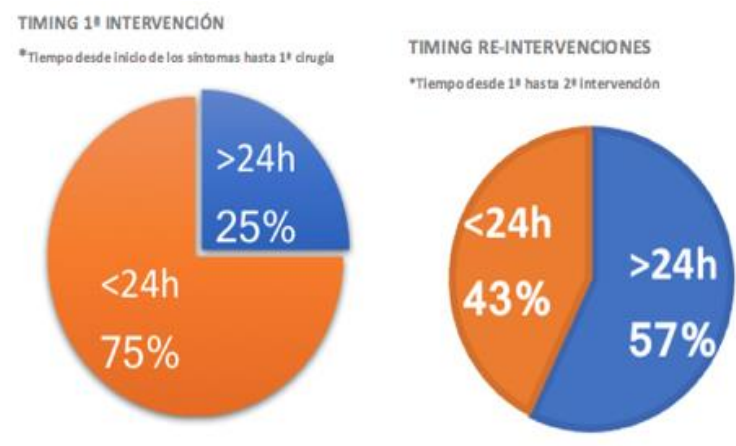

\section{BIBLIOGRAFÍA}

1. McHenry CR, Piotrowski JJ, Petrinic D, Malangoni MA. Determinants of mortality for necrotizing soft-tissue infections. Ann Surg 1995; 221(5):558-63.

2. Wong $\mathrm{CH}$, Khin LW, Heng KS et al. The LRINEC (laboratory risk indicator for necrotizing fasciitis) score: a tool for
El presente trabajo cuenta con todas las limitaciones inherentes a cualquier estudio retrospectivo que trata una serie de casos y, además, el tamaño de la muestra es limitado consistiendo en 8 pacientes. En este sentido es conveniente recalcar que el estudio no pretende arrojar datos de significación estadística. Sin embargo, dada la baja incidencia, consideramos que se trata de una serie valiosa a partir de la cual analizar el manejo de esta patología en nuestro medio.

Como conclusión, a la hora de optimizar los resultados en el manejo de la FN intentando mejorar la supervivencia, existen 2 puntos clave: El primero es el diagnóstico precoz, que sólo es posible si conocemos la clínica, siendo importante identificar el prototipo de paciente de riesgo para despertar la alarma. No existen muchas herramientas diagnósticas objetivas que apoyen el diagnóstico actualmente, salvo algunas pruebas de imagen como el TC, pero que nunca se deben realizar si van a suponer un retraso en el tratamiento de un paciente con un cuadro clínico compatible. El segundo punto clave es realizar una intervención temprana y programar las re-intervenciones de antemano para cumplir los tiempos propuestos.

distinguishing necrotizing fasciitis from other soft tissue infections. Crit Care Med 2004; 32: 1,535-1,541

3. Wong $\mathrm{CH}$, Chang $\mathrm{HC}$, Pasupathy $\mathrm{S}$, Khin LW, Tan JL, Low CO. Necrotizing fasciitis: clinical presentation, microbiology, and determinants of mortality. J Bone Joint Surg Am 2003; 85:1454-60.

4. Parra Caballero P., Perez Esteban S., Patiño Ruiz ME y cols. Revisión Actualización en 
fascitis necrotizante. Semin Fund Esp Reumatol 2012; 13:41-8.

5. May AK. Skin and soft tissue infections. Surg Clin North Am 2009; 89:403- 420

6. Angoules AG. Necrotising fasciitis of upper and lower limb: a systematic review. Injury. 2007 Dec;38 Suppl 5:S19-26. Epub 2007 Nov 28.

7. Bisno, A. L., \& Stevens, D. L. (1996). Streptococcal Infections of Skin and Soft Tissues. New England Journal of Medicine, 334(4), 240-246.

8. Stevens DL, Bryant AE. Necrotizing SoftTissue Infections. N Engl J Med 2017; 377:2253

9. Anaya DA, Dellinger EP. Necrotising softtissue infection: diagnosis and management. Clin Infect Dis 2007;44:705-10.

10. Tantraworasin, A., Khamnuan, P., Chongruksut, W., Jearwattanakanok, K., Patumanond, J.(2015). Necrotizing fasciitis: epidemiology and clinical predictors for amputation. International Journal of General Medicine Volume 8, 195-202.

11. Miller LG, Perdreau-Remington F, Rieg G et al. Necrotizing fasciitis caused by community-associated methicillin-resistant Staphylococcus aureus in Los Angeles. N Engl J Med 2005; 352: 1,445-1,453.

12. Tsitsilonis $\mathrm{S}$, Druschel $\mathrm{C}$, Wichlas $\mathrm{F}$ et al. Necrotizing fasciitis: is the bacterial spectrum changing? Langenbecks Arch Surg 2013; 398: 153-159

13. Bucca K, Spencer R, Orford N et al. Early diagnosis and treatment of necrotizing fasciitis can improve survival: an observational intensive care unit cohort study. ANZ J Surg 2013; 83: 365-370.

14. Glass, G., Sheil, F., Ruston, J., Butler, P.(2015). Necrotising soft tissue infection in a UK metropolitan population. The Annals of The Royal College of Surgeons of England 97(1), 46-51.
15. Baharestani MM. Negative pressure wound therapy in the adjunctive management of necrotizing fasciitis: examining clinical outcomes. Ostomy Wound Manage. 2008;54:44-50.

16. Lee JY, Jung $H$, Kwon $H$, Jung $S N$. Extended negative pressure wound therapyassisted dermatotraction for the closure of large open fasciotomy wounds in necrotizing fasciitis patients. World J Emerg Surg 2014;9:29. 